\title{
ASSISTIVE TECHNOLOGIES FOR PAIN MANAGEMENT IN AMPUTEES: A REVIEW
}

\author{
Kamiar Ghoseiri ${ }^{1 *}$, Mostafa Allami², Mohammad Reza Soroush ${ }^{2}$, Mohammad Yusuf Rastkhadiv $^{3}$
}

${ }^{1}$ Department of Orthotics and Prosthetics, School of Rehabilitation Sciences, Hamadan University of Medical Sciences,

Hamadan, Iran.

${ }^{2}$ Janbazan Medical and Engineering Research Center (JMERC), Tehran, Iran.

${ }^{3}$ Department of Occupational Therapy, School of Rehabilitation Sciences, Hamadan University of Medical Sciences, Hamadan, Iran.

*Email: Kamiar_g@yahoo.com

DOI: https://doi.org/10.33137/cpoj.v1i2.32008

\section{INTRODUCTION}

The prevalence of limb amputation is increasing globally as a devastating experience that can physically and psychologically affect the lifestyle of a person. The residual limb pain and phantom limb pain are common disabling sequelae after amputation surgery. Assistive devices/technologies can be used to relieve pain in people with amputation. The present review aimed to introduce the existing assistive devices/technologies for pain management in people with amputation.

\section{METHODS}

An electronic literature search was performed in three databases of PubMed, ISI Web of Science, and Scopus to find articles that directly or indirectly reported pain management using assistive devices/technologies in people with amputation. All articles were evaluated by two independent reviewers to extract their demographic and methodological characteristics. Moreover, the type of assistive device/technology and pain relief level were extracted for all articles.

\section{RESULTS}

The electronic search found 142 articles in the three chosen databases. After excluding duplicate and irrelevant articles, and those incompatible with review criteria, 9 articles were remained for evaluation and data extraction.

\section{CONCLUSION}

The present review revealed there are few available assistive devices/technologies for pain management in people with amputation. The majority of the available assistive devices/technologies relieve pain conservatively. However, some of them are working by infusing a medication and some needs surgery to place some components inside body. There is a great need to portable, wireless, smart, and thin devices/technologies to stimulate the spinal cord and peripheral nerves by electrical, thermal, mechanical, or pharmaceutical stimulus. The overall pain relief cannot easily be compared among assistive devices/technologies in this review due to different study designs, interventions, and characteristics of participants. Long-term randomized clinical trials are required to evaluate the effectiveness of available assistive devices/technologies. Although some preliminary efforts have been done to resolve post amputation pain, more attention from researchers, clinicians, designers, engineers and manufacturers are required.

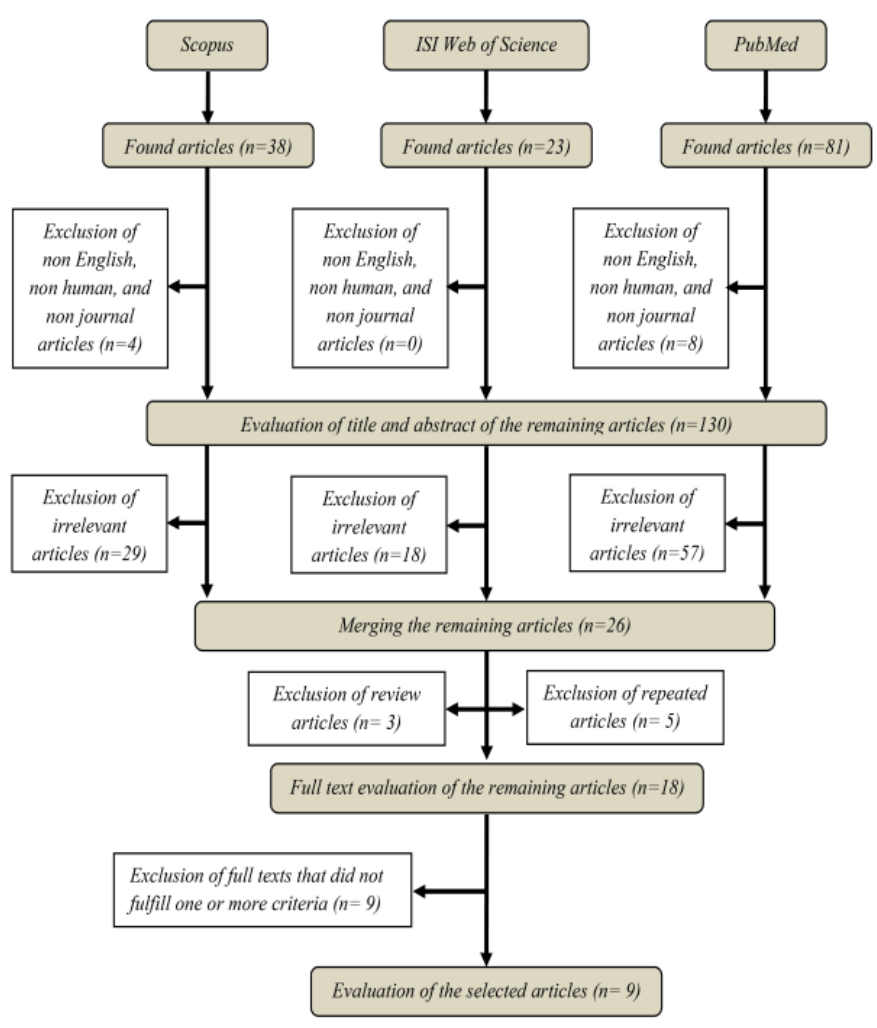

Figure 1. The procedure for selection of studies from three databases. 


\section{SIGNIFICANCE}

- Residual limb pain and phantom limb pain are common complaints of people with amputation.

- There are few available assistive devices/technologies to relieve residual limb pain and phantom limb pain.

- More attention to resolve post amputation pain is required from researchers, clinicians, designers, engineers and manufacturers

\section{REFERENCES}

1. Dijkstra PU, et al. Phantom pain and risk factors: a multivariate analysis. J Pain Symptom Manage. 2002; 24, 578-

85. https://doi.org/10.1016/S0885-3924(02)00538-9

2. Ephraim PL, et al. Phantom pain, residual limb pain, and back pain in amputees: results of a national survey. Arch Phys Med Rehabil. 2005; 86, 1910-9. DOI: 10.1016/j.apmr.2005.03.031

3. Pet MA, et al. Does targeted nerve implantation reduce neuroma pain in amputees? Clin Orthop Relat Res. 2014; 472, 2991-3001. DOI: $10.1007 / \mathrm{s} 11999-014-3602-1$

\section{DISCLOSURE}

The authors report no declarations of interest. However, the financial support of the research was done by the Janbazan Medical and Engineering Research Center (JMERC).

\section{ACKNOWLEDGMENTS}

The authors would like to sincerely thank the financial support of the Janbazan Medical and Engineering Research Center (JMERC), Tehran, Iran for this material. 